MODELING, IDENTIFICATION AND CONTROL, 1995, VOL. 16, NO. 4, 177-191

doi:10.4173/mic.1995.4.1

\title{
Computational fluid dynamics simulation of bioreactors
}

\author{
B. H. HJERTAGER and K. MORUD
}

Keywords: Bioprocess, modeling, multiphase, chemical reactor

Multi-dimensional models of flow processes in bioreactors are presented. Particular emphasis is given to models that use the two-fluid technique. The models use a two-equation turbulence model and a Monod type kinetic reaction model. Predictions are given for both bubble column and mechanically stirred reactors.

\section{Introduction}

\subsection{Background and motivation}

Many production processes based on biotechnology depend on oxygen-requiring microorganisms in suspension. Gas-liquid bioreactors from the core of all such processes. A bioreactor designer has to ensure that the rector provides for: (1) adequate oxygen transfer, (2) sufficient agitation and mixing and (3) can handle the heat transfer loads, at acceptable capital and operating costs.

The oxygen needs are often the most difficult to satisfy. In theory, there should be no difficulty in meeting these demands, were it not for the special constraints encountered in bioprocessing:

the oxygen solubility in the typical aqueous media is low

the biological broths can be highly viscous and have non-Newtonian behaviour the low shear tolerance and susceptibility to mechanical damage of the biological catalyst

These limitations often lead to poor mixing and mass transfer in bioreactors. Furthermore, the restrictions on acceptable shear levels are becoming more tight with increasing use of cell cultures in commercial production.

Reliable assessments of performance and scale-up of bioreactors will require more detailed understanding and characterisation beyond the scope of traditional empirical approaches in process design, i:e. global performance correlations obtained in replica modeling tests. The research needed to achieve this goal will involve experimental investigations and mathematical modeling of the local process encountered by organisms flowing through the non-homogeneous turbulent and bubbly flow fields.

\subsection{Previous work}

The 2D and 3D modeling of bioreactors has only just started. At the 2nd Int. Conference on Bioreactor Fluid Dynamics, held at Cambridge, England, 21-23 Sept.

Received 15 September 1994.

Telemark Institute of Technology (HiT-TF) and Telemark Technological R\&D Centre (Tel-Tek) Kjølnes, N-3914 Porsgrunn, Norway. An early version of this paper was presented at the Bioreactor Performance Symposium.
Helsingør, Denmark, 15-17 March 1993 . 
1988, the Chairman of the conference, Dr. Roger King, opened the meeting by stating that true process models had to take account of the interaction between fluid dynamics and reaction kinetics in a $2 \mathrm{D}$ or $3 \mathrm{D}$ space. Of the 25 papers presented at the conference only three papers discussed application of detailed models of bioreactor fluid dynamics. The paper by Trägårdh (1988) presented a 2D model of a mechanically stirred reactor. The bubble flow was described by a simplified model. The paper by Boysan et al. (1988) also applied a 2D model to an agitated vessel, whereas the model of Smith et al. (1988) presented preliminary results from a 3D model and single phase approximation of an agitated vessel with baffles. Trägårdh et al. (1990) has further developed his model by introducing a two-scale turbulence model. Issa and Gosman (1981), Bakker and van der Akker (1991) and Gosman et al. (1992) have presented calculations of the 3D processes that occur in the agitated vessel. Norman et al. $(1993,1994)$ have introduced integrated models in which both the fluid dynamics and microbial kinetics is modeled.

The flow pattern in bubble columns has been analysed by Torvik and Svendsen (1990) and Morud et al. (1991) using two-dimensional computational fluid dynamics models. The results of these studies showed reasonable agreement between model predictions and experiments. The model of Morud and Hjertager (1992) has also included submodels for the biochemical reactions that occur in bubble column bioreactors.

It is clear from the status of these works that there is still room for improvements, regarding both modeling the transport and reaction process as well as geometry modeling.

\subsection{Objective of the paper}

The aim is to present 2D/3D prediction/design models for the chemical reaction and transport processes of stress-sensitive organisms in multi-phase flows of mechanically stirred and bubble-driven bioreactors.

Emphasis will be given to models of the finite-domain type in which the governing equations are integrated over discrete volumes inside the reactor. A multi-fluid approach will be described to enable modeling of the processes that occur in the liquid and gas phases as well as the transfer between the phases. Models of turbulence and agitators will also be discussed. Finally, some examples of flow modeling of bioreactors will be given.

\section{General considerations}

\subsection{Model types}

Models of bioreactors have until recently been limited to zero-dimensional models. In these models total and species mass balances have been set up with the whole reactor volume as control surface. The balances include terms containing accumulation, net inflow and new generation rate of the species in question. The main assumption in this model is that there are no gradients in the dependent variables (i.e. concentrations). No momentum balances are needed for this type of model. The consequence of this model is that a set of ordinary differential equations have to be solved. It is only during the last decade that 2D and 3D models have been developed. These multi-dimensional models set up balance equations for sub-domains (computational cells or control volumes) in the reactor volume. As for the zero-dimensional models total and species mass balances have to be set up for each of these sub-domains. In addition, the flow in and out of the sub-domains must also he calculated. For this we need to put up 
momentum balances. One momentum balance has to be set up for each direction, i.e. two for $2 \mathrm{D}$ and three for $3 \mathrm{D}$. The new process that has to be included is diffusion of the conserved quantity in question. This is due to the fact that concentrations are not homogeneous inside the bioreactor. This means that in addition to accumulation, net inflow and net generation, net diffusion needs to be modeled. In order to model this diffusion, gradient transport is assumed with an effective transport coefficient (diffusion coefficient for species transport and viscosity for momentum transport). Since the flow is turbulent this effective transport coefficient is dependent on characteristics of the turbulence. Two parameters are decisive for describing the state of turbulence, namely local turbulence velocity and length scale of the turbulent eddies. This has led to the development of the so-called two-equation turbulence models (Launder and Spalding (1974)). These models are deduced by setting up conservation equations for two quantities. The most popular of these models are named the $k-\varepsilon$ model, where $k$ is the turbulent kinetic energy and $\varepsilon$ is the rate of dissipation of turbulent kinetic energy. From $k$ and $\varepsilon$ we may deduce the turbulence velocity and length scale and from them the effective transport coefficient. A general feature of the multi-dimensional models is that we need to solve a set of partial differential equations. This means that special care must be taken to obtain efficient means of solving these equations (Patankar (1980), Spalding (1985)). Special treatments are needed for impeller and baffle regions in mechanically stirred bioreactors.

\subsection{Impeller}

The impeller region has been modeled in different ways. Harvey and Greaves (1982) calculated turbulent single phase flow in an agitated vessel with baffles. They used a 2-D description and the standard $k-\varepsilon$ turbulence model and specified $k, \varepsilon$ and velocity at the impeller surface. The swirling motion of fluid on the impeller boundary is assumed to be solid body motion. Some predictions of mean flow and turbulence were compared with experimental data. Placek et al. (1986) have predicted and measured the flow patterns and turbulence properties in a 2-D baffled vessel. They applied a 3-equation turbulence model and assumed anisotropic turbulence in the impeller region. The model requires proper assumptions concerning the impeller boundary, thus they got different results depending on the assumptions at the impeller boundary. Ju et al. (1990) compute the flow field of a 3-D calculation domain using a nonisotropic viscosity turbulence model. They use a tangential jet boundary condition that require knowledge of three experimental parameters and assume zero gradient of $k$ and $\varepsilon$ at the impeller periphery. Kresta and Wood (1991) use a swirling radial jet mode, and the parameters needed for its application are the peak discharge velocity and flow angle at the impeller tip. Based on experimental results available in this region, optimum parameters are suggested. Pericleous and Patel (1987) use a simple 2-D approach with the impeller treated as a time-averaged source/sink quantity in the appropriate momentum equation.

\subsection{Baffles}

The presence of baffles further complicates the calculations. Harvey and Greaves (1982) and Pericleous and Patel (1987) model the baffle by introducing a drag force on the swirling component of the flow. When a 3-D description is used, the baffles do not need to be modeled, but are included as a part of the calculation domain. 


\section{Governing equations}

\subsection{General}

Two different approaches are available for setting up the governing equations for two-phase flow in bioreactors. One method is the so-called PSIC (Particle-Source-InCell) procedure originally presented by Migdal and Agosta (1967). This method treats the continuous phase (liquid) in a usual Eulerian description, whereas the dispersed phase (bubbles) are described in a Lagrangian way. This means that bubbles are tracked through the flow domain from inlet to outlet. The presence of bubbles are coupled to the liquid phase through sources of mass and momentum. This method may only be used for small values of the volume fraction of the gas bubbles.

The other method deduce the governing equations based on the Eulerian concept and are named the two-fluid method (Spalding 1985). This means that the phases are treated as interpenetrating fluids that share the space and interact with each other through the source terms. All equations are such that the volume fractions may take values between zero and one. The remainder of this paper will present a two-fluid model.

\subsection{The two-fluid model}

The numerical model of the turbulent two-phase flow is based on a two fluid model (Spalding (1985)). Transport equations governing the conservation of mass, momentum and scalar variables like turbulent quantities $(k$ and $\varepsilon$ ) and concentration of chemical species are derived for each phase. The problem is formulated in cylindrical polar coordinates. The flow is assumed to be axi-symmetrical, so that the equations reduce to two dimensions. The two-fluid conservation equations governing the mean turbulent flow field are written as follows:

Mass:

$$
\frac{\partial}{\partial t}(\alpha \rho)_{k}+\frac{\partial}{\partial x}(\alpha \rho U)_{k}+\frac{1}{r} \frac{\partial}{\partial r}(\alpha r \rho V)_{k}=0
$$

\section{Momentum:}

$$
\begin{aligned}
\frac{\partial}{\partial t}\left(\alpha \rho U_{i}\right)_{k}+\frac{\partial}{\partial x}\left(\alpha \rho U U_{i}\right)_{k}+\frac{1}{r} \frac{\partial}{\partial r}\left(\alpha r \rho V U_{i}\right)_{k}= & -\frac{\partial}{\partial x}\left(\alpha \sigma_{i x}\right)_{k}-\frac{1}{r} \frac{\partial}{\partial r}\left(\alpha r \sigma_{i r}\right)_{k} \\
& +\alpha_{k} \sigma_{\theta \theta} r^{(1-i)}+\alpha_{k} \frac{\partial p}{\partial x_{i}}+F_{i, k}+\alpha_{k} \rho_{k} g_{i}
\end{aligned}
$$

Scalar:

$$
\frac{\partial}{\partial t}(\alpha \rho \phi)_{k}+\frac{\partial}{\partial x}(\alpha \rho U \phi)_{k}+\frac{1}{r} \frac{\partial}{\partial r}(\alpha r \rho V \phi)_{k}=-\frac{\partial}{\partial x}\left(\alpha J_{\phi, x}\right)_{k}-\frac{1}{r} \frac{\partial}{\partial r}\left(\alpha r J_{\phi, r}\right)_{k}+S_{\phi}
$$

here the subscript $k$ refers to the gas $(g)$ or liquid $(l)$ phase, and the subscript $i$ refers to the axial $(i=1)$ or radial $(i=2)$ coordinate-direction. The pressure is considered to be common for the two phases, and both gas and liquid is assumed to be incompressible. Turbulent diffusive transport of momentum and scalar variables are denoted $\sigma_{i j}$ and $J_{\Phi, j}$ respectively. Viscous effects are assumed to be negligible. 


\subsection{Interfacial friction}

The interfacial friction term, for example in the axial direction, is given by

$$
F_{x, k}= \pm C \alpha_{g} \alpha_{l}\left(U_{g}-U_{l}\right)
$$

where $C=5 \cdot 10^{4} \mathrm{~kg} \mathrm{~m}^{-3} \mathrm{~s}^{-1}$. This formulation was applied by Schwarz and Turner (1988) calculating the fluid dynamics of gas stirred baths. The value of the constant corresponds to a slip velocity between the gas and liquid phases of $0.2 \mathrm{~m} / \mathrm{s}$. This is a good approximation to the experimentally determined terminal velocity in water of single air bubbles of diameter between 1 and $10 \mathrm{~mm}$. The slip velocity is almost constant over this range of diameters because of increasing non-sphericity. For this reason equation (4) is claimed to give a much better representation than does the formula for drag on a sphere.

Amarasooriya and Theofanous (1988) give a more rigorous model description of the interfacial drag force, splitting it into one part effected by friction and another part effected by mass. The flow is divided into flow regimes; homogeneous bubble flow $(\alpha<0.3)$, heterogeneous bubble flow $(0.3<\alpha \leqslant 0.7)$ and droplet flow $(\alpha>0.7)$, and different formulations of the drag coefficient and bubble diameters are given for each flow regime.

\subsection{Interfacial mass transfer}

The interphase mass transfer is taken account of through the source terms as follows:

$$
\left.S_{m_{j, l}}=\left(k_{l} \cdot a\right)_{l}\right) \cdot\left(m_{j, g}-m_{j, l}\right)
$$

The $\left(k_{l} a\right)$ is related to the dissipation rate, $\varepsilon$, the local fraction, $\alpha$ and bubble diameter, $d_{b}$ (Trägårdh (1988)).

\subsection{Turbulence model}

The Reynolds stresses, $\sigma_{i j}$, in equation (2) and the turbulent scalar fluxes in equation (3) are related to the mean flow field through the Boussinesq approximation and are given by

$$
\sigma_{x x}=-2 \mu_{t}\left(\frac{\partial U}{\partial x}\right) ; \quad \sigma_{r r}=-2 \mu_{t}\left(\frac{\partial V}{\partial r}\right) ; \quad \sigma_{\theta \theta}=-2 \mu_{t}\left(\frac{V}{r}\right) ; \quad \sigma_{x r}=-\mu_{t}\left(\frac{\partial U}{\partial r}+\frac{\partial V}{\partial x}\right)
$$

and

$$
J_{\phi, x}=-\frac{\mu}{\sigma_{\phi}} \frac{\partial \phi}{\partial x} ; \quad J_{\phi, r}=-\frac{\mu_{t}}{\sigma_{\phi}} \frac{\partial \phi}{\partial r}
$$

The Prandtl/Schmidt number $\sigma_{\phi}$ is assumed to be constant, and the turbulent viscosity $\mu_{t}$ is modeled using the two parameter $k-\varepsilon$ turbulence model (Launder and Spalding (1974)) as

$$
\mu_{t}=c_{\mu} \rho_{k} \frac{k^{2}}{\varepsilon}
$$

For two-phase flows, the turbulence is commonly assumed to be a property of the continuous (liquid) phase (Torvik and Svendsen (1990)). The modeled transport equations for the turbulent kinetic energy $k$ and the dissipation tate $\varepsilon$ is therefore solved 


\begin{tabular}{ccccc}
\hline$c_{\mu}$ & $c_{1}$ & $c_{2}$ & $\sigma_{k}$ & $\sigma_{\varepsilon}$ \\
\hline 0.09 & 1.44 & 1.92 & 1.0 & 1.3 \\
\hline
\end{tabular}

Table 1. Values of constants in the $k-\varepsilon$ model.

only for the liquid phase:

$$
\begin{aligned}
\frac{\partial}{\partial t}(\alpha \rho k)_{l}+\frac{\partial}{\partial x}(\alpha \rho U k)_{l}+\frac{1}{r} \frac{\partial}{\partial r}(\alpha r \rho V k)_{l}= & \frac{\partial}{\partial x}\left(\alpha \frac{\mu_{t}}{\sigma_{k}} \frac{\partial k}{\partial x}\right)_{l} \\
& +\frac{1}{r} \frac{\partial}{\partial r}\left(\alpha r \frac{\mu_{t}}{\sigma_{k}} \frac{\partial k}{\partial r}\right)_{l}+\alpha_{l}\left(G+P_{b}-\rho \varepsilon\right)_{l} \\
\frac{\partial}{\partial t}(\alpha \rho \varepsilon)_{l}+\frac{\partial}{\partial x}(\alpha \rho U \varepsilon)_{l}+\frac{1}{r} \frac{\partial}{\partial r}(\alpha r \rho V \varepsilon)_{l}= & \frac{\partial}{\partial x}\left(\alpha \frac{\mu_{t}}{\sigma_{\varepsilon}} \frac{\partial \varepsilon}{\partial x}\right)_{l} \\
& +\frac{1}{r} \frac{\partial}{\partial r}\left(\alpha r \frac{\mu_{t}}{\sigma_{\varepsilon}} \frac{\partial \varepsilon}{\partial r}\right)_{l}+\alpha_{l} \frac{\varepsilon_{l}}{k_{l}}\left(c_{l}\left(G+P_{b}\right)-c_{2} \rho \varepsilon\right)_{l}
\end{aligned}
$$

The production of turbulence due to mean strain is given by:

$$
G=\mu_{t}\left\{2\left[\left(\frac{\partial U}{\partial x}\right)^{2}+\left(\frac{\partial V}{\partial r}\right)^{2}+\left(\frac{V}{r}\right)^{2}\right]+\left(\frac{\partial U}{\partial r}+\frac{\partial V}{\partial x}\right)^{2}\right\}
$$

Additional turbulent kinetic energy is produced due to the work induced by the bubbles when they move through the liquid phase:

$$
P_{b}=c_{i} F_{x, l}\left(U_{\mathrm{g}}-U_{l}\right)
$$

Although the influence of bubbles on turbulence production is uncertain we have chosen to follow Lopez de Bertodano et al. (1990) where a value of 0.02 was used for $c_{i}$, which means that about 2 per cent of the bubble-induced turbulence goes into the large eddy structure of the continuous phase.

The values of the other constants applied in the $k-\varepsilon$ model are given in table 1 .

\subsection{Stirred tank modifications}

The flow in the stirred tank may be calculated using a 2-D model description which neglects gradients in the tangential direction. The equations used for computations of agitated flow is mainly as given above, but additional equations and terms are introduced. The tangential velocity, $w$, is calculated as a scalar variable as in equation 3. Additional terms appear on the right hand side of the momentum equations:

Radial momentum:

$$
-2\left(\frac{\alpha \mu_{t} v}{r^{2}}\right)_{k}+\left(\frac{\alpha \rho w^{2}}{r}\right)_{k}
$$

Axial momentum:

$$
-\frac{2}{r} \frac{\partial}{\partial r}\left(\alpha \mu_{t} r w\right)_{k}
$$

A constant interfacial drag term is included in the tangential direction in a similar manner as for the two other directions, see equation 4 . 


\begin{tabular}{lll}
\hline Conservation of & $\Phi$ & $S_{\Phi}$ \\
\hline Yeast & $X$ & $\mu_{s} X$ \\
Substrate & $S$ & $-q_{S} X$ \\
Ethanol & $E$ & $\left(q_{\left.e p-q_{e c}\right) X}\right.$ \\
\hline
\end{tabular}

Table 2. Source terms of chemical species.

The Rushton impeller may, as a first approximation modeled as a rigid body motion with constant source term in the equation of tangential velocity. A more refined model is given by Pericleous and Patel (1987). This model introduces the influence of the Rushton impeller by an additional source term in the tangential momentum equation as:

$$
S_{i m p, w_{k}}=\frac{1}{2} \cdot \rho_{k}\left[(\omega \cdot r)^{2}-w_{k}^{2}\right] \cdot C_{f} \cdot n \cdot A_{f}
$$

Where $C_{f}$ is the drag coefficient of a flat plate perpendicular to a stream of fluid, $n$ is the number of paddles on the turbine, and $A_{f}$ is the volume averaged paddle area. The value for $C_{f}$ is taken as 2.0. $\omega$ is the angular velocity of the impeller.

The baffles are modeled according to Pericleous and Patel (1987). Also in this situation an additional source is included in the tangential momentum equation as:

$$
S_{b, w_{k}}=-\frac{1}{2} \cdot \rho_{k} w_{k}^{2} \cdot C_{b} \cdot n \cdot A_{b}
$$

where $C_{b}$ is the drag coefficient, $n$ is the number of baffles and $A_{b}$ is the volume averaged area of the baffles.

Production of turbulent kinetic energy in equation 11 is extended to include turbulent production due to the tangential velocity:

$$
G=\mu_{t}\left\{2\left[\left(\frac{\partial U}{\partial x}\right)^{2}+\left(\frac{\partial V}{\partial r}\right)^{2}+\left(\frac{V}{r}\right)^{2}\right]+\left(\frac{\partial U}{\partial r}+\frac{\partial V}{\partial x}\right)^{2}+\left(r \frac{\partial}{\partial r}\left(\frac{w}{r}\right)\right)^{2}+\left(\frac{\partial w}{\partial x}\right)^{2}\right\}
$$

\subsection{Kinetic model}

The kinetic model of the fermentation, adapted from the research group at KTH (Enfors et al. (1992)), is based on Monod's model and include ethanol formation. No oxygen limitation is assumed in this model and therefore the transport of oxygen is not calculated. The transport equations for chemical species in the form given in equation 3 are needed, with the source terms given in table 2, where

$$
\begin{aligned}
& q_{s}=q_{s \max } * S /\left(S+K_{s}\right) \\
& q_{o s}=q s^{*} Y_{o s} \\
& q_{e p}=q_{s x f} * Y_{e s} \\
& \text { if } q_{o s}>q_{o \max } \text { then } q_{o s}=q_{o \max } \\
& q_{s x a}=q_{o s} / Y_{o s} \\
& q_{e c}=\left(q_{o \max }-q_{o s}\right) / Y_{o e} * E /\left(E+K_{s e}\right) \\
& q_{o}=q_{o s}+q_{e c}{ }^{*} Y_{o e} \\
& \mu=\mathrm{q}_{s x a} * Y_{x s a}+q_{s x f} * Y_{x s f}+q_{e c} * Y_{x e}
\end{aligned}
$$

and $q_{\text {smax }}=1.4 \mathrm{hr}^{-1}, K_{s}=0.2 \mathrm{~g} / 1, Y_{o s}=0.5, q_{o \max }=0.3 \mathrm{hr}^{-1}, Y_{e s}=0.35, Y_{o e}=0.8$, $K_{s e}=0.5 \mathrm{~g} / 1, Y_{x s a}=0.5, Y_{x s f}=0.15, Y_{x e}=0.8$.

\subsection{Boundary conditions}

Inlet and outlets are easily defined by specifying velocities and relevant conditions for the other dependant variables. For the liquid phase, the large gradients near the wall are treated by wall functions similar to those established for single phase flow (Launder and Spalding (1974)). 


\section{Numerical procedure}

A two fluid model is used to determine the flow field, where the gas and liquid phase are handled as spacesharing interspersed continua. The partial differential equations are expressed by algebraic so called 'finite domain' equations by dividing the calculation domain into several control volumes and by integration over these control volumes for each phase with respect to time and space. The discretizations are done by using interpolation functions, here the upwind scheme described in the book of Patankar (1980) is used for space, and a fully implicit scheme for time.

The momentum equations are coupled through the drag terms and when solving the equations sequentially, the convergence may be slow for the large drag terms. This is avoided by using the partial elimination algorithm (PEA). Point iteration has been used to solve the volume fraction and momentum equations.

The equations are solved sequentially, and because of the nonlinearities and coupling between the equations there is a need for an iterative solution procedure. The single phase solution algorithm SIMPLE (Semi-Implicit Method for Pressure Linked Equations) by Patankar Spalding (1972) extended for two phase flow by Spalding (1985) has been used.

A summary of the algorithm involves the following points:

(1) Initialize dependent variables.

(2) Update. Apply boundary conditions.

(3) Solve the volume fraction equations with modified expressions for the continuity equations Jacobi iteration.

(4) Solve the momentum equations with the PEA algorithm and the latest available solution for the pressure. Jacobi iteration.

(5) Solve the pressure correction equation with Gauss-Seidel line iteration (TDMA algorithm).

(6) Repeat from 2 until a specified convergence criterium is satisfied.

(7) Solve other equations, like concentrations and turbulent quantities.

(8) If more timesteps, goto 2.

\section{Simulations}

Simulations using the model presented above are performed for two different types of bioreactor types, namely, the bubble column reactor and the mechanically agitated reactor. Bubble column reactors are characterized by the fact that agitation is performed by the air that is supplied at the bottom of the reactor, whereas in the mechanically agitated reactors the stirring is accomplished by a rotating stirrer placed on a shaft.

\subsection{Bubble column}

Lab. scale. Measurements of number averaged gas bubble velocity have been performed in an air-water bubble column using Laser doppler/Phase doppler anemometer (LDA/PDA) (Davidsen (1991)). The column had an internal diameter of $0.15 \mathrm{~m}$ and a height of $2 \mathrm{~m}$. The air was supplied at the bottom through a porous distributor plate that covers the whole tube area. The calculated and measured air velocity across the diameter is shown in fig. 1 for four different superficial air velocities. As we can see the agreement is best for the highest air velocity. The general trend is well predicted, namely high upward gas velocity in the centre of the column and low downward gas flow close to the wall. Figure 2 shows the measured and calculated 


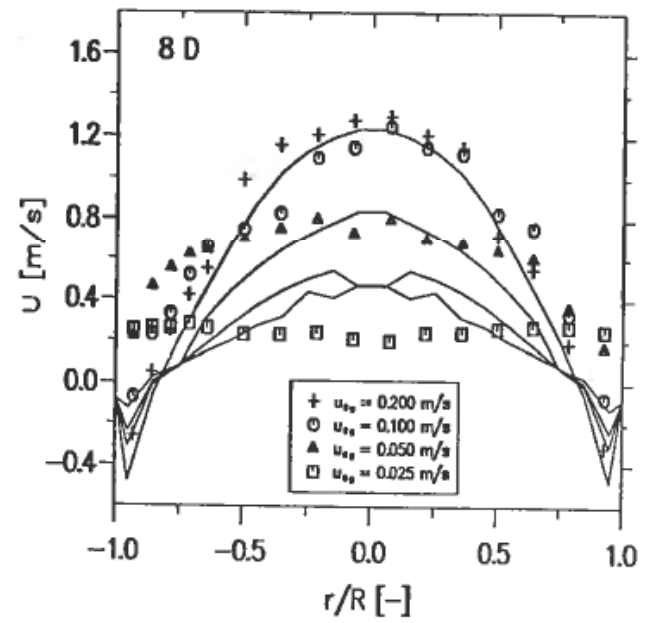

Figure 1. Axial gas velocities as function of dimensionless radius.

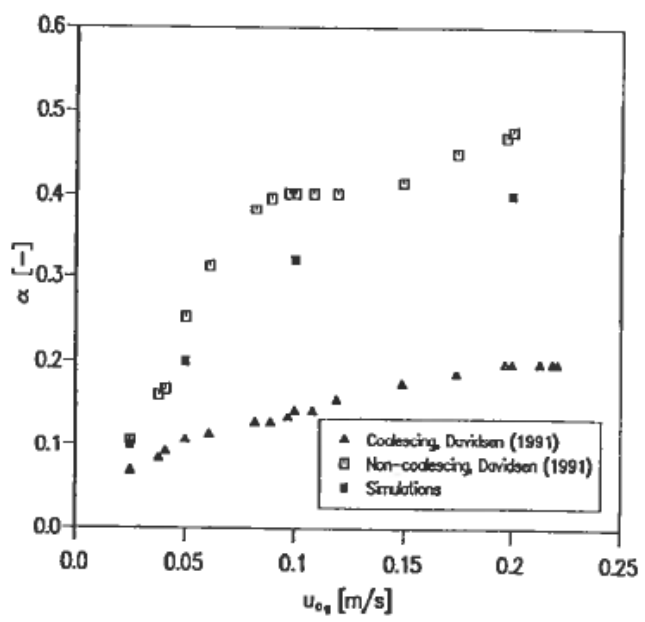

Figure 2. Total void fraction as function of superficial gas velocity.

average void fraction in the column as function of the superficial gas velocity. Experiments have been carried out using both coalescing and non-coalescing systems. The calculated void fraction compares well with the data for non-coalescing system.

Yeast fermentor. Calculations of flow pattern and biokinetic reaction have been performed for a large scale bubble column for Baker's yeast fermentation located at Jästbolaget, Sweden (George et al. (1992)). The fermentor is $17,5 \mathrm{~m}$ high with a diameter of $4.0 \mathrm{~m}$ and has an initial liquid volume of $90 \mathrm{~m}^{3}$. There is one feed point $1 \mathrm{~m}$ above the bottom. The air is supplied evenly at the bottom of the reactor. Both the air and the sugar is supplied with varying flow rate. The inlet sugar concentration is $380 \mathrm{~g} / \mathrm{l}$ and the initial concentrations are: sugar: $0.05 \mathrm{~g} / \mathrm{l}$, yeast: $10 \mathrm{~g} / \mathrm{l}$, ethanol $0.0004 \mathrm{~g} / \mathrm{l}$. Additional assumptions are:

The air and sugar supply are assumed constant; $0.08 \mathrm{~m} / \mathrm{s}$ (superficial) and 2900 1/hr respectively.

Liquid density is set to $110 \mathrm{~g} / \mathrm{l}$. 
The process time is $10 \mathrm{hr}$.

No oxygen limitations in the fermentor, and thereby no mass transfer of oxygen between the two phases needs to be calculated.

A stationary flow field has been computed. This flow field has then been used as a basis of computing the transport, production and consumption of the species involved. Figure 3 shows the simulated streamlines of gas. It is interesting to note that in this large scale simulation the flow pattern is different than in the small scale air-water system mentioned above. In the fermentor the air flow upward along the wall and downward in the centre. The void fraction is between 0.34 and 0.40 . Simulated sugar concentration distribution $6 \mathrm{~h}$ after the start of the fermentation is given in Fig. 4. Obviously the concentration is largest close to the inlet.

Jästbolaget, Sweden, have measured sugar concentrations near the wall at the height of $2.5 \mathrm{~m}, 6.5 \mathrm{~m}$ and $12.5 \mathrm{~m}$ during the whole fermentation period. The simulated values are plotted versus time in Fig. 5. Simulated ethanol concentration is also given in Fig. 5 together with simulated yeast concentrations. The concentrations of ethanol and yeast are the same in the entire liquid volume. Yeast concentration increases monotonically for the fermentation period. The ethanol concentration peaks at about three hours. The experimental data shows that there are gradients in the sugar inside the reactor, but the magnitude is smaller than the predictions. The reasons for this discrepancy is difficult to assess, but it is important that more local flow measurements are performed as well as improving the biokinetic model.

\subsection{Stirred reactor}

Measurements of number averaged gas bubble velocity in a 15 litre stirred reactor has been carried out using a Laser-doppler anemometer. The computations are performed on a numerical mesh comprising 47 grid cells in the axial direction and 24 grid cells in the radial direction. The air is considered to enter at the bottom of the vessel through a porous plate with radius $2 \mathrm{~cm}$ and with blockage of $99 \%$.

The streamlines of liquid and gas are shown in Fig 6 and 7 with a flow rate of $7.5 \mathrm{l} / \mathrm{min}$. The liquid contours show that the impeller cause the liquid to move radially towards the wall and to give two circulation loops. For the gas streamlines the picture is not the same as for the liquid. The gas bubbles are subject to inertia which makes the two phases flow differently. The gas flow has several circulation loops. The two strongest are above and below the impeller. The gas fraction distribution is shown in Fig. 8. It is seen that the peak gas fraction in the liquid is located in the 'eye' of the two gas vorticies. Figure 9 shows the calculated and measured radial gas velocity just outside the impeller for three different rpm. As we can see from the figure the agreement is very good. It is especially gratifying to see that the measured maximum in gas velocity outside the tip of the impeller is well predicted.

\section{Concluding remarks}

A computation method for turbulent two-phase flow and reaction kinetics in 2D bioreactors has been presented. Flow and kinetics of a production scale Bakers yeast fermentation in a bubble column have been computed. The simulated concentrations at three positions in the column have been compared with experiments performed by Jästbolaget, Sweden. Experimental and computational results of flow pattern in a 15 litre stirred vessel have been presented. Further emphasis should be made on establishing more experimental data, to improve and continue the model work and to 


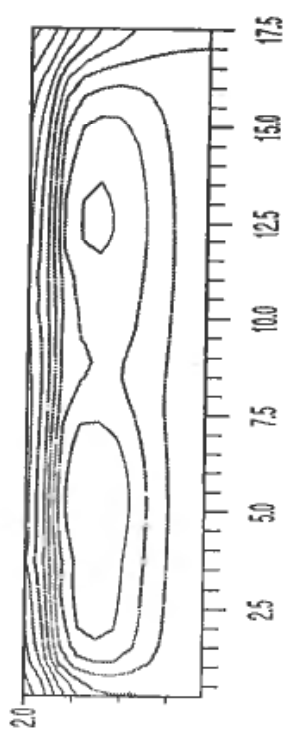

Figure 3. Streamlines gas.

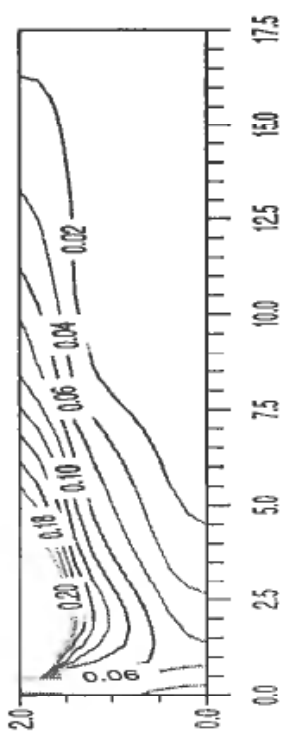

Figure 4. Sugar concentrations $[\mathrm{g} / \mathrm{l}]$.

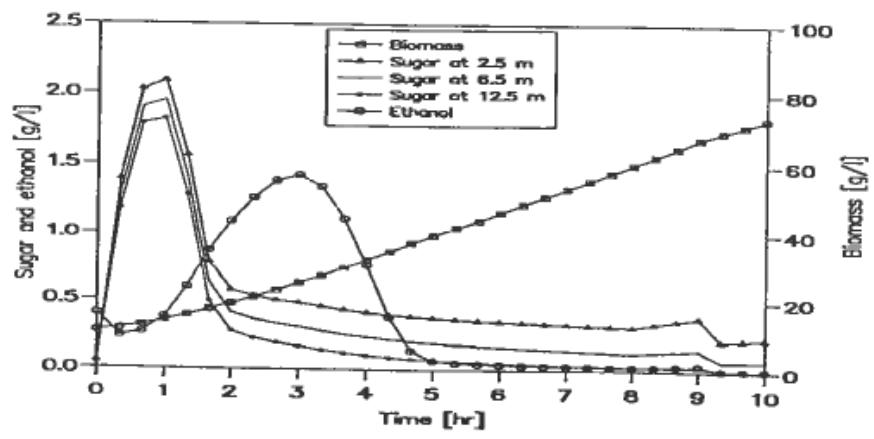

Figure 5. Sugar, ethanol and biomass concentrations during fermentation. 


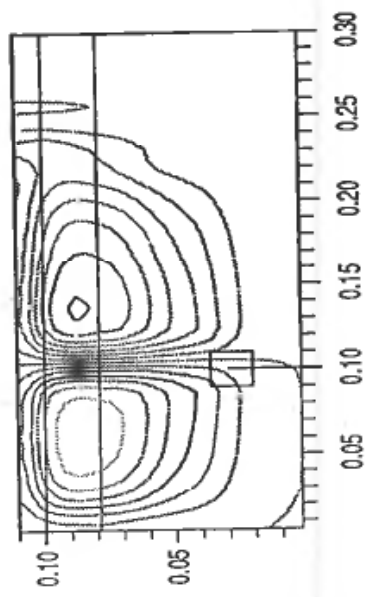

Figure 6. Streamlines liquid.

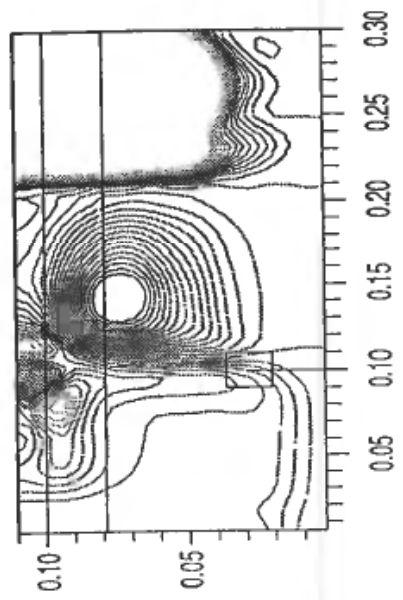

Figure 7. Streamlines gas.

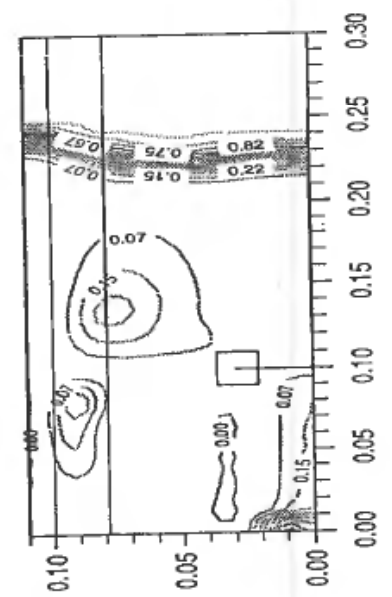

Figure 8. Void fraction. 


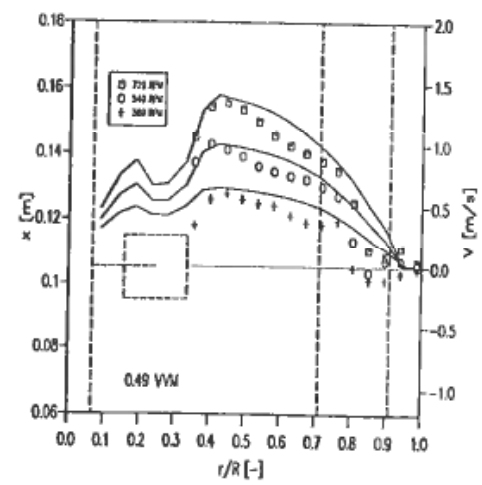

Figure 9. Radial gas velocities.

verify the simulated results against the experimental data. The biokinetic model should be extended to include effect of oxygen limitation in the reaction rate and take account of the distribution of dissolved oxygen (DO). The flow model should be extended to include the effects of non-Newtonian fluids.

\section{ACKNOWLEDGEMENTS}

The project is supported by a grant from the Nordic Programme on Bioprocess Engineering under the auspices of NI, the Nordic Fund for Technology and Industrial Development and the Research Council of Norway. The authors are grateful to the following MSc students at Telemark Institute of Technology that have contributed to the work: M. Davidsen, I. Grøtberg.

\section{REFERENCES}

Amarasooriya, W. H. and Theofanous, T. G. (1980). Premixing of steam explosions. A three-fluid model, Preprint (University of California, Santa Barbara).

BAKKER, A. and VAN DER AKKER, H. E. (1991). A computational study on dispersing gas in a stirred reactor, Proceedings of the European Congress on Mixing, pp. 199-208.

BOYSAN, F. et al. (1988). The growth of Cathanranthus roseus in stirred tank bioreactors, in Bioreactor Fluid Dynamics (Elsevier Applied Science Publ.) pp. 245-258.

DAVIDSEN, M. (1991). Analysis of flow pattern in a bubble column MSc Thesis (in Norwegian), Telemark Institute of Technology.

EnFors, S. O., George, S. and LaRSON, G., KTH (1992). Private communications.

Gosman, A. D., LeKaKou, C., Poltris, S., IsSA, R. I. and LoONEY, M. K. (1992). Multidimensional modeling turbulent two-phase flows in stirred vessels, AIChE Journal, 38, 1946-1956.

GeORGE, S., LARSSON, G., ENFORS, S. O., MORUD, K. and HJERTAGER, B. H. (1992). Large-scale test of hydrodynamic-microbial model predictability and comparison with scale down reactor. $215 \mathrm{~m}^{3}$ bubble column at Svenska Jästfabrik $\mathrm{AB}$, Sollentuna, Sweden, Joint interim report TEL-TEK/KTH.

HARVEY, P. S. and GREAVES, M. (1982). Turbulent flow in an agitated vessel, Trans. Inst. of Chem. Eng., 60, 195-210.

IsSA, R. I. and GoSMAN, A. D. (1981). The computation of three-dimensional turbulent two-phase flows in mixer vessels, in Numerical methods in laminar and turbulent flow, pp. 827-838.

JU, S. Y., MULVAHILL, T. M. and PIKE, R. W. (1990). Three-dimensional turbulent flow in agitated vessels with a nonisotropic viscosity turbulence model, Canadian J. Chem. Eng., 68, 3-16.

KRESTA, S.M. and WOOD, P. (1991). Prediction of the three-dimensional turbulent flow in stirred tanks, AIChE Journal, 37, 448-460.

LaI, K. Y. and SAlCUDEAN, M. (1987). Computer analysis of multi-dimensional turbulent buoyancy-induced two phase flows in gas-agitated reactors, Computers and Fluids,
13, 215-295. 
LAUNDER, B. F. and SPALDING, D. B. (1974). The numerical computation of turbulent flows, Computer Methods in Applied Mechanics and Engineering, 3, 269-289.

LOPEZ DE BERTODANO, M., LeE, S.-J., LAHEY, R. T. JR. and DREW, D. A. (1990). The prediction of two-phase distribution phenomena using a Reynolds stress model, Journal of Fluids Engineering, 112, 107.

Migdal, D. and AGostA, V. D. (1967). A source flow model for continuum gas-particle flow. Appl. Mech., 35, 860-865.

Morud, K., SOlBerg, T. and HJeRTAGer, B. H. (1991). Turbulent two-phase air water flow in a bubble column, Paper XI in Proc. of the Bioprocess Engineering meeting in Sandnes, 2-4 April (Biotechnology Research Foundation, Lund, Sweden).

MoRUd, K. and HJerTAGer, B. H. (1992). Multidimensional modelling of processes in bioreactors: Flow and biochemical reaction in a bubble column and flow in a stirred vessel. Proc. of the Bioprocess Engineering meeting in Stockholm, pp. 40-57 (Biotechnology Research Foundation, Lund, Sweden).

Norman, H. Enfors, S.-O, HJertager, B. H., Larsson, G., Morud, D., TrägÅrdh, C. and TøRNKVIST, M. (1993). Verification of integrated microbial and fluid dynamics: Saccharomyces cerevisiae production on $30 \mathrm{~m}^{3}$ scale, in Progress in Biotechnology (Elsevier, Amsterdam, Holland), Vol. 9, Part II, pp. 935-938.

Norman, H., Morud, K., HJertager, B. H., TräGÅrdH, C., LARSSON, G. and EnFors, S.-O. (1994). CFD modelling and verification of flow and conversion in a $1 \mathrm{~m}^{3}$ bioreactor, 3rd Int. Conference on Bioreactor and Bioprocess Fluid Dynamics, Cambridge, UK, 14-16 September (MEP, London, UK), pp. 241-258.

Patankar, S. V. (1980). Numerical Heat Transfer and Fluid Flow (McGraw-Hill).

PATANKaR, S. V. and SPALding, D. B. (1972). A calculation procedure for heat, mass and momentum transfer in three-dimensional parabolic flows, Int. Journal of Heat and Mass Transfer, 15, 1787-1800.

Placek, J., TAVlaRIDES, L. L., SMIth, G. W. and FORT, I. (1986). Turbulent flow in stirred tanks Part II: A twoscale model of turbulence, AIChE Journal, 32, 1771-1786.

Pericleous, K. A. and PATEL, M. K. (1987). The source and sink approach in the modelling of stirred reactors, PCH PhysicoChemical Hydrodynamics, 12, 279-97.

RANADE, V. V. and JOSHI, J. B. (1990). Flow generated by a disc turbine: Part Il-Mathematical modelling and comparison with experimental data, Trans IChemE, 68, 34-50.

SChWARZ, M. P. and TURNER, W. J. (1988). Applicability of the Standard $k-\varepsilon$ Turbulence Model to Gas Stirred Baths, Appl. Math. Modelling, 12, 273-279.

SMITH, T. J. and ReILlY, C. D. (1988). Predictions of the flow in fermentors and implications for scale-up, in Bioreactor Fluid Dynamics (Elsevier Applied Science Publ.), pp. 431-441.

SPALDING, D. B. (1985). Computer simulation of two-phase flows with special reference to nuclear reactor systems, in Computational Techniques in Heat Transfer, Eds: R. W. Lewis, K. Morgan, J. A. Johnson and W. R. Smith, (Pineridge Press, Swansea, UK), pp. 1-44.

TORVIK, R. and SVENDSEN, H. F. (1990). Modelling of slurry reactors. A fundamental approach Chem. Eng. Sci., 45, 2325-2332.

TRÄGARDH, C. (1988). A hydrodynamic model for the simulation of an aerated agitated fed-batch fermentor, in Bioreactor Fluid Dynamics (Elsevier Applied Science Publ.), pp. 117-134.

TRÄGÅRDH, C., HEINZLE, E. and SANER, E. U. (1990). Mathematical modelling of mixing and biokinetics in agitated tank fermentors, Proc. of Fifth European Conference on Biotechnology, pp. 797-800.

\section{Nomenclature}

$a$ interfacial surface area

$A_{b}$ volume averaged surface area of baffles

$A_{f}$ volume averaged surface area of paddles

$B$ width of impeller blade

$C$ drag coefficient

$D_{i}$ impeller diameter

$D_{t}$ tank diameter

$F_{k}$ interface friction force 
$g$ gravitational acceleration

$G$ production of turbulent kinetic energy

$J$ flux

$k$ turbulent kinetic energy

$K_{s}$ saturation constant for substrate

$K_{s e}$ saturation constant for ethanol

$n$ number of baffles or paddles

$p$ pressure

$p_{b}$ production of turbulent kinetic energy due to bubbles

$q_{s}$ spec. substrate concentration rate

$q_{s \max }$ max. spec. substrate concentration rate

$q_{s x a}$ substrate consumption rate, aerobe

$q_{s x f}$ substrate consumption rate, anerobe

$q_{e p}$ ethanol production rate

$q_{e c}$ ethanol consumption rate

$q_{o}$ total oxygen consumption rate

$q_{o s}$ oxygen consumption rate

$q_{\text {omax }}$ max. spec. oxygen concentration rate

$S$ source term

$t$ time

$u, v$ fluctuating velocity

$U, V$ mean velocity

$W$ height of impeller blade

$Y_{o s}$ yield $\mathrm{gO} / \mathrm{gS}$

$Y_{\text {es }}$ yield $\mathrm{gE} / \mathrm{gS}$

$Y_{x s a}$ yield $\mathrm{gX} / \mathrm{gS}$, aerobic

$Y_{x s f}$ yield $\mathrm{gX} / \mathrm{gS}$, anaerobic

$Y_{x e}$ yield $\mathrm{gX} / \mathrm{gE}$

\section{Greek letters}

$\alpha$ void fraction

$\varepsilon$ dissipation of turbulent kinetic energy

$\varphi$ fluctuating quantity

$\Phi$ general variable

$\mu$ viscosity

$\mu_{s}$ specific growth rate

$\rho$ fluid density

$\sigma$ shear stress

\section{Subscripts}

$g$ gas phase

$k$ phase number

$l$ liquid

$t$ turbulent 